\title{
Effect of temozolomide on the viability of musculoskeletal sarcoma cells
}

\author{
YUTA KUSABE $^{1}$, HIROYUKI KAWASHIMA $^{2}$, AKIRA OGOSE$^{2}$, TARO SASAKI $^{2}$, \\ TAKASHI ARIIZUMI ${ }^{3}$, TETSUO HOTTA ${ }^{2}$ and NAOTO ENDO ${ }^{2}$
}

\author{
${ }^{1}$ School of Medicine; ${ }^{2}$ Division of Orthopedic Surgery, Niigata University Graduate School of Medical and Dental Sciences, \\ Niigata 851-8510; ${ }^{3}$ Department of Orthopedic Surgery, Niigata Cancer Center Hospital, Niigata 951-8566, Japan
}

Received September 16, 2014; Accepted June 16, 2015

DOI: $10.3892 / 01.2015 .3506$

\begin{abstract}
Musculoskeletal sarcomas (MSS) are a heterogeneous group of malignancies with relatively high mortality rates. The prognosis for patients with MSS is poor, with few drugs inducing measurable activity. Alkylating agents, namely ifosfamide and dacarbazine, which act nonspecifically on proliferating cells, are the typical therapy prescribed for advanced MSS. A novel alkylating agent, temozolomide (TMZ), has several advantages over existing alkylating agents. TMZ induces the formation of $O^{6}$-methylguanine in DNA, thereby inducing mismatches during DNA replication and the subsequent activation of apoptotic pathways. However, due to conflicting data in the literature, the mechanism of TMZ action has remained elusive. Therefore, the present study aimed to evaluate apoptosis in MSS cells treated with $\mathrm{TMZ}$, and to evaluate the correlation between TMZ action and survival pathways, including the phosphoinositide 3-kinase (PI3K)/Akt and extracellular signal-regulated kinase (ERK)1/2 mitogen activated protein kinase (MAPK) pathways. Cell proliferation was evaluated by performing an XTT (sodium 3'-[1-(phenylaminocarbonyl)-3,4-tetrazolium]-bis (4-methoxy-6-nitro) benzene sulfonic acid hydrate) assay. Apoptotic morphological changes, for example chromatin condensation, were evaluated by fluorescence confocal microscopy. The expression of the apoptosis-associated proteins caspase-3, poly adenosine diphosphate ribose polymerase (PARP), Akt and ERK1/2, was determined by western blotting. The results of the present study indicated that, in certain MSS cells, the $\mathrm{IC}_{50}$ value was lower than that in TMZ-sensitive U-87 MG cells. Furthermore, TMZ treatment was associated with apoptotic morphological changes
\end{abstract}

Correspondence to: Dr Hiroyuki Kawashima, Division of Orthopedic Surgery, Niigata University Graduate School of Medical and Dental Sciences, 1-757 Asahimachi-dori, Niigata 951-8510, Japan

E-mail: inskawa@med.niigata-u.ac.jp

Key words: temozolomide, musculoskeletal sarcoma, apoptosis, phosphoinositide 3-kinase/Akt and the expression levels of pro-apoptotic cleaved caspase-3 and PARP were also increased in TMZ-treated MSS cells. In addition, the results indicated that PI3K/Akt and ERK1/2 MAPK were constitutively phosphorylated in MSS cells, and phosphorylation of PI3K/Akt was suppressed in certain cells, and maintained in other cells, by TMZ. These observations emphasized the plasticity of MSS cells, and suggested that this plasticity may contribute to the variance in cell sensitivity to TMZ and TMZ-resistance in MSS.

\section{Introduction}

Temozolomide (TMZ; 3,4-dihydro-3-methyl-4-oxoimidazo[5,1-d]-1,2,3,5-tetrazin-8-carboxamide), a 3-methyl analogue of mitozolomide, is an imidazotetrazine class methylating agent. Similarly to dacarbazine (DTIC), the active metabolite of TMZ is the linear triazine monomethyl triazenoimidazole carboxamide (MTIC) (1). DTIC requires initial activation in the liver, while TMZ is metabolized to MTIC in the peripheral blood at physiological $\mathrm{pH}$. The subsequent degradation of MTIC to the highly reactive methyldiazonium ion results in the formation of toxic methyl adducts with DNA bases. The methyldiazonium ion promotes cytotoxicity via $O^{6}$-methylation of guanine, leading to base-pair mismatch, inhibition of DNA replication and subsequent cell cycle arrest and apoptosis (2). TMZ resistance has been ascribed to the removal of methyl groups by $O_{6}$-methylguanine-DNA-methyltransferase. Furthermore, in vitro and human xenograft studies have demonstrated a broad spectrum of TMZ activity in murine tumors (2).

Musculoskeletal sarcomas (MSS) are a heterogeneous group of malignant neoplasms, which are derived from the connective tissue. Sarcomas represent $\sim 1 \%$ of cancers in adults. MSS may occur anywhere in the body; however, the lower extremities represent the most common site of appearance, followed by the upper extremities, trunk, retroperitoneum and the head and neck area (3). MSS treatment has markedly improved in recent years, owing to a greater understanding of the local growth characteristics of tumors, novel computed tomography (CT) and magnetic resonance imaging (MRI) technology for the evaluation and diagnosis of solid tumors, as well as the development of multidisciplinary treatments that facilitate local tumor treatment and functional tissue 
preservation (4). Surgical resection is the primary treatment strategy for MSS; however, chemotherapy has become more common for treatment of the majority of bone sarcomas, and may have potential benefits for the treatment of patients with soft-tissue sarcomas (STS). Numerous patients that present with locally advanced disease may require multimodal therapy, including chemotherapy and chemo-radiation. Doxorubicin and ifosfamide are typically recommended as the first-line systemic treatment in unresectable and/or metastatic STS. However, despite an initial anti-tumor response, numerous patients gradually develop resistance to these therapies. Salvage therapy options following the failure of frontline MSS chemotherapy are limited; thus, novel, more effective agents are required for the successful treatment of these diseases.

Administration of TMZ alone or in combination with other chemotherapeutic agents has demonstrated activity in patients with pretreated MSS, particularly among patients with certain histological subtypes, for example leiomyosarcoma, solitary fibrous tumors and Ewing's sarcoma (5-9). However, the enhancement in patient survival following treatment with TMZ is not significant. Thus, elucidating the mechanism of TMZ action against MSS is essential. Therefore, the present study aimed to determine the effect of TMZ on cell viability in MSS in order to identify histological subtypes that may be suitable for TMZ-based treatment.

\section{Materials and methods}

Cell lines and cell culture. Sarcoma cell lines, including the NOS1 osteosarcoma cell line (10), NMS-2 malignant peripheral nerve sheath tumor cell line (11) and NEPS epithelioid sarcoma cell line (12), were established under the approval of the Institutional Review Board of Niigata University Hospital (Niigata, Japan). The HS-SY-II synovial sarcoma cell line was a gift from Dr H. Sonobe (Department of Pathology, Kochi Medical School, Kochi, Japan) (13), the SYO-1 synovial sarcoma cell line was gifted by Dr A. Kawai (Department of Orthopedic Surgery, Okayama University, Okayama, Japan) (14), the 402-92 liposarcoma cell line was a gift from Dr P. Åman (Department of Clinical Genetics, University Hospital, Lund, Sweden) (15), the ASPS-KY alveolar soft part sarcoma cell line was a gift from Dr S. Yanoma (Kanagawa Cancer Center, Yokohama, Japan) (16) and the FU-EPS-1 (12) and SFT-8606 (17) epithelioid sarcoma cell lines were gifts from Dr H. Iwasaki (Department of Pathology, Fukuoka University School of Medicine, Fukuoka, Japan). The aforeentioned cell lines underwent 50-100 passages and screening tests confirmed no mycoplasma infection. The remaining cell lines used in the present study were obtained from commercial sources, and all cell lines used are outlined in Table I. NOS1, Saos-2, NMS-2, 402-92, ASPS-KY, HT-1080, SFT-8606, FU-EPS-1 and NEPS cells were maintained in RPMI-1640 (Invitrogen Life Technologies, Carlsbad, CA, USA). U-87 MG, HS-SY-II and SYO-1 cells were maintained in Dulbecco's modified Eagle's medium (Invitrogen Life Technologies). The SKNMC cells were maintained in $\alpha$-minimum essential medium (Invitrogen Life Technologies). All media were supplemented with $10 \%$ fetal bovine serum (FBS; PAA Laboratories $\mathrm{GmbH}$,
Pasching, Austria) containing 1\% antibiotics and antimycotics (penicillin, streptomycin and amphotericin B; Invitrogen Life Technologies). All cell cultures were incubated at $37^{\circ} \mathrm{C}$ in an atmosphere containing $5 \% \mathrm{CO}_{2}$ with $100 \%$ humidity.

Effect of TMZ on cell viability and morphology. All cell lines were plated on 96 -well plates $\left(\sim 3.5 \times 10^{3}\right.$ cells/well $)$ and incubated for $24 \mathrm{~h}$. Subsequently, cells were washed with medium and incubated with fresh medium (control) or medium containing $125,250,500$ or $1000 \mu \mathrm{M}$ TMZ. Cell viability was assessed using the XTT (sodium 3'-[1-(phenylaminocarbonyl)-3,4-tetrazolium]-bis (4-methoxy-6-nitro) benzene sulfonic acid hydrate) assay according to the manufacturer's instructions, following exposure to the various concentrations of TMZ for $120 \mathrm{~h}$ at $37^{\circ} \mathrm{C}$. Subsequently, the plates were incubated for $4 \mathrm{~h}$ at $37^{\circ} \mathrm{C}$ under the same conditions as the previous 120 -h incubation. Absorbance was measured at $490 \mathrm{~nm}$ against a reference wavelength of $655 \mathrm{~nm}$ using a microplate reader (model 680; Bio-Rad Laboratories, Inc., Hercules, CA, USA). The cell viability (\%) and $\mathrm{IC}_{50}$ value $(\mu \mathrm{M})$ were calculated relative to the controls at $100 \%$, and the mean $\mathrm{IC}_{50}$ value was calculated from three experiments experiments using CurveExpert software (version 1.3; Daniel G. Hyams, Madison, AL, USA). Subsequent to the 120 -h incubation but prior to the 4-h incubation, cell morphology was observed daily using an Olympus phase-contrast microscope ULWCD 0.30 (IMT2; Olympus Corp., Tokyo, Japan) and photomicrographs of the control wells and $1000 \mu \mathrm{M}$ TMZ wells were taken at a magnification of $\mathrm{x} 40$.

Western blot analysis of apoptosis and cell survival/proliferation pathways. Cultured cells were harvested and subjected to western blot analysis to assess the induction of apoptosis via the phosphorylation status of phosphoinositide 3-kinase (PI3K)/Akt and extracellular signal-regulated kinase (ERK)1/2 mitogen activated protein kinase (MAPK), and the cleavage of caspase- 3 and poly (adenosine diphosphate-ribose)-polymerase (PARP). The cells were plated on 60 -mm culture dishes at a density of $0.3-5.0 \times 10^{5}$ cells/dish $24 \mathrm{~h}$ prior to treatment with $250 \mu \mathrm{M}$ TMZ. At 24,48 and $72 \mathrm{~h}$ post-exposure, the cells were subjected to western blot analysis to determine the expression levels of each of the aforementioned proteins. Briefly, the cells were washed twice with ice-cold phosphate-buffered saline (PBS) and suspended in SDS-PAGE running buffer comprised of: $62.5 \mathrm{mM}$ Tris, $\mathrm{pH} 6.8 ; 2 \%$ SDS; $5 \%$ glycerol and $6 \mathrm{M}$ urea (Invitrogen Life Technologies). Cell lysates were passed through a 23 -gauge needle (Terumo, Tokyo, Japan) ten times, and the protein concentration of the supernatant was measured by bicinchoninic acid assay (Pierce Biotechnology, Inc.; Thermo Fisher Scientific, Rockford, IL, USA). Subsequently, dithiothreitol $(50 \mathrm{mM})$ and bromophenol blue $(0.025 \%)$ (Sigma-Aldrich, St. Louis, MO, USA) were added, and the samples were boiled for $5 \mathrm{~min}$ at $95^{\circ} \mathrm{C}$ and stored at $-80^{\circ} \mathrm{C}$. Equal quantities of protein were subjected to $7 \%$ SDS-PAGE and then transferred to a nitrocellulose membrane (GE Healthcare Life Sciences, Little Chalfont, UK). The membrane was probed with primary antibodies against caspase-3 (rabbit polyclonal; cat no. 9662; 1:1,000), PARP (rabbit polyclonal; cat no. 9542; 
Table I. Summary of human cell lines used in the present study.

\begin{tabular}{lll}
\hline Name & \multicolumn{1}{c}{ Cell origin } & \\
\hline SKNMC & Ewing's sarcoma & Source \\
Saos-2 & Osteosarcoma & ATCC \\
NOS1 & Osteosarcoma & ATCC \\
NMS-2 & Malignant peripheral nerve sheath tumor & Niigata University, Niigata, Japan \\
NEPS & Epithelioid sarcoma & Niigata University \\
FU-EPS-1 & Epithelioid sarcoma & Niigata University \\
SFT-8606 & Epithelioid sarcoma & Dr H. Iwasaki \\
HS-SY-II & Synovial sarcoma & Dr H. Iwasaki \\
SYO-1 & Synovial sarcoma & Dr H. Sonobe \\
HT-1080 & Fibrosarcoma & Dr A. Kawai \\
$402-92$ & Liposarcoma & Health Science Research Resources Bank, Osaka, Japan \\
ASPS-KY & Alveolar soft part sarcoma & Dr P. Aman \\
U-87 MG & Glioblastoma & Dr S. Yanoma \\
\hline
\end{tabular}

ATCC, American Type Culture Collection, Manassas, VA, USA.

1:1,000), pT202/Y204-MAPK (rabbit polyclonal; cat no. 9101; 1:1,000), total MAPK (rabbit polyclonal; cat no. 9102; 1:1,000), pS473-AKT (rabbit polyclonal; cat no. 9271; 1:1,000), total AKT (rabbit polyclonal; cat no. 9272; 1:1,000; Cell Signaling Technology, Inc., Danvers, MA, USA), and actin (mouse monoclonal; cat no. A2228; 1:3,000; Sigma-Aldrich), and then with horseradish peroxidase-conjugated IgG donkey anti-rabbit (cat no. NA934; 1:3,000) or sheep anti-mouse (cat no. NA931; 1:3,000) secondary antibodies (GE Healthcare Life Sciences). The bands were visualized by enhanced chemiluminescence (ECL Western Blotting Detection kit; GE Healthcare Life Sciences).

Statistical analysis. All data are expressed as the mean \pm standard deviation. The differences between groups were evaluated using a two-tailed Student's t-test and all statistical analysis was performed using Microsoft Excel software (Microsoft Corporation, Redmond, WA, USA). $\mathrm{P}<0.01$ was considered to indicate a statistically significant difference.

\section{Results}

Cell growth is inhibited in TMZ-treated MSS cells. As shown in Fig. 1, TMZ reduced the viability of all tumor cells in a dose-dependent manner following incubation for $120 \mathrm{~h}(\mathrm{P}<0.01)$. However, the $\mathrm{IC}_{50}$ value of $\mathrm{TMZ}$ varied between cell lines. In U87-MG cells, the $\mathrm{IC}_{50}$ was $348 \mu \mathrm{M}$, whereas the $\mathrm{IC}_{50}$ value of TMZ in SKNMC, NOS1, HS-SY-II, SYO-1 and 402-92 cells was $<300 \mu \mathrm{M}$. By contrast, the $\mathrm{IC}_{50}$ value of TMZ in HT1080, NMS-2, Saos-2, ASPS-KY, NEPS, FU-EPS-1 and SFT-8606 cells was $>366 \mu \mathrm{M}$. Based on these results, TMZ inhibited the proliferation of SKNMC, NOS1, HS-SY-II, SYO-1 and 402-92 cells more potently than that of U87-MG cells. By contrast, HT1080, NMS-2, Saos-2, ASPS-KY, NEPS, FU-EPS-1 and SFT-8606 cells were more resistant to TMZ than $\mathrm{U} 87-\mathrm{MG}$ cells.
TMZ induces morphological changes in MSS cells. Photomicrographs of TMZ-treated cells were obtained using phase-contrast microscopy. Cell death was observed $120 \mathrm{~h}$ post-exposure to $1000 \mu \mathrm{M} \mathrm{TMZ}$, as indicated by a mass of rounded, floating, highly refractive cells. Such cell death was observed in 11 of the 13 cell lines evaluated (Fig. 2). Notably, no cell lysis was observed in the FU-EPS-1 or ASPS-KY cells $\left(\mathrm{IC}_{50}>1000 \mu \mathrm{M}\right)$.

TMZ induces apoptosis in all cell lines. Following treatment with TMZ, all 13 cell lines were examined for evidence of apoptosis by western blot analysis, using caspase-3 and PARP antibodies (Fig. 3). Activation of caspase-3 via protein cleavage has a critical role in apoptosis (18). Furthermore, the proteolytic cleavage of PARP by activated caspase- 3 serves as a more prominent marker of apoptosis (19). The results of the present study indicated that pro-caspase-3 and PARP levels were reduced, while cleaved caspase-3 and PARP were upregulated. These data indicated that TMZ induced caspase activation and proteolysis of caspase-3 and PARP in MSS cells. In particular, a marked enhancement in caspase-3 cleavage was observed at 48 and $72 \mathrm{~h}$ following TMZ exposure in the SKNMC, Saos-2, NOS1, HS-SY-II, HT1080, 402-92 and NEPS cells.

Effects of PI3K/Akt and ERK1/2 MAPK on MSS cell survival and proliferation. The initiation of apoptosis is dependent on the DNA repair system, as well as on multiple signaling pathways, including the phosphorylation status of PI3K/Akt and ERK1/2 MAPK. These signaling pathways were evaluated via western blot analysis. The results indicated that endogenous Akt was characterized by the phosphorylation of Ser473 (Fig. 4). Furthermore, the basal levels of p-Akt were dose-dependently reduced in SYO-1, HT-1080, NMS-2 and ASPS-KY cells following treatment with $250 \mu \mathrm{M}$ TMZ for $24 \mathrm{~h}$. Typically, p-Akt is constitutively expressed in U-87 MG cells $(20,21)$. Although treatment with $\mathrm{TMZ}$ initially reduced $\mathrm{p}$-Akt 

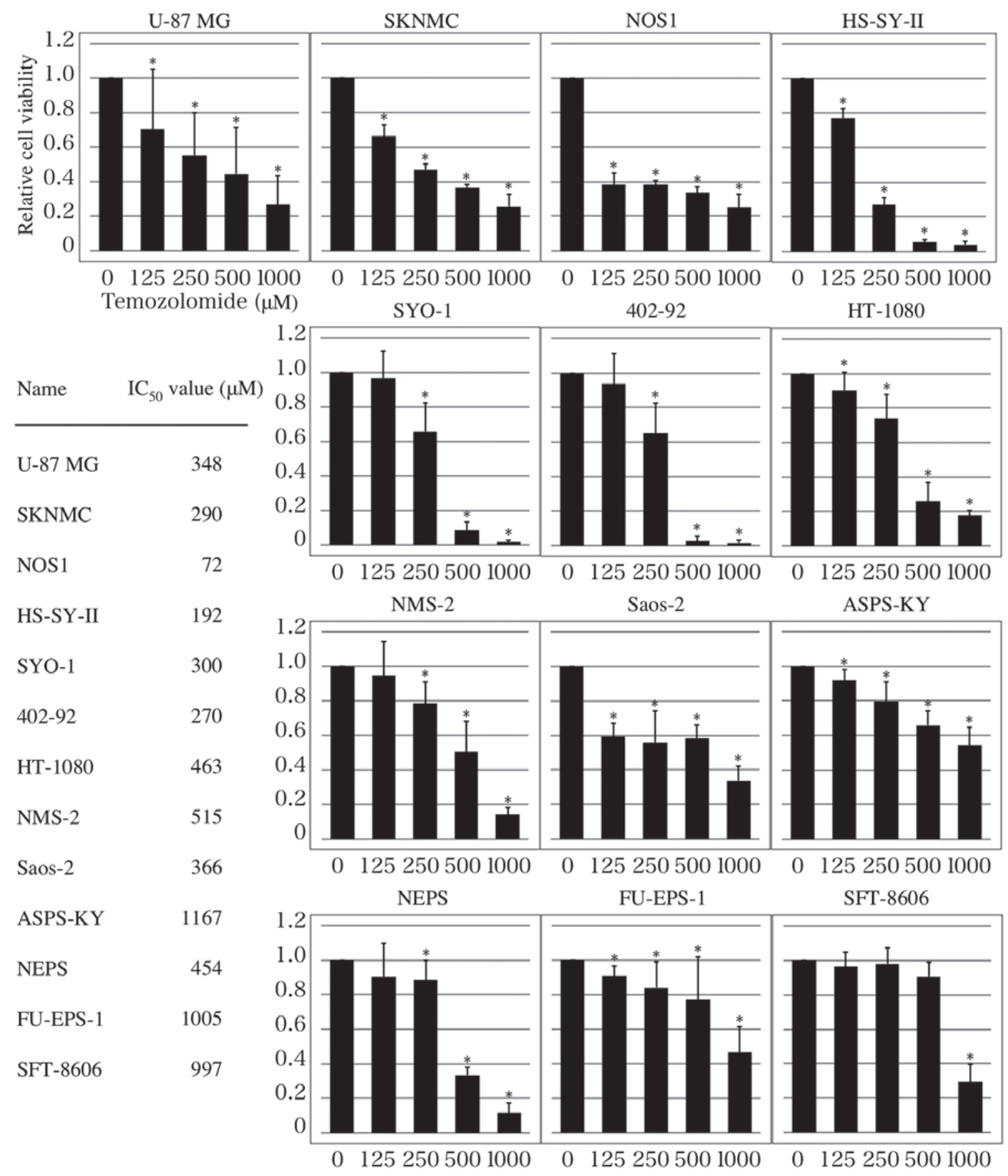

Figure 1. TMZ inhibits MSS cell growth. Inhibitory effects of TMZ on MSS growth were determined using an XTT assay. Cell viability $(\%)$ and $\mathrm{IC}_{50}$ values $(\mu \mathrm{M})$ were calculated relative to the controls at $100 \%$, and the mean $\mathrm{IC}_{50}$ value was calculated from three replicates. The data represents the mean of three experiments. Based on these results, TMZ inhibited the growth of SKNMC, NOS1, HS-SY-II, SYO-1 and 402-92 cells more potently than U-87 MG cells. By contrast, HT1080, NMS-2, Saos-2, ASPS-KY, NEPS, FU-EPS-1 and SFT-8606 cells were more resistant to TMZ than U-87 MG cells. "P<0.01 vs. non-treated control group. TMZ, temozolomide; MSS, musculoskeletal sarcoma.

expression, p-Akt markedly increased at $72 \mathrm{~h}$. We hypothesze that whilst constitutive activation of this pathway is emerging as a marker of sensitivity to TMZ, the activity of TMZ may be limited by the presence of feedback loops, leading to the reactivation of $\mathrm{p}-\mathrm{AKT}$. The activation status of ERK1/2 MAPK was examined by evaluating the expression of ERK1/2 by western blotting. The results indicated that there was basal activation of ERK1 and 2 in the MSS cells. In the presence of $250 \mu \mathrm{M}$ TMZ, the levels of p-ERK1/2 were marginally decreased in ASPS-KY and NEPS cells following $48 \mathrm{~h}$ treatment.

\section{Discussion}

The activity of TMZ in patients with glioblastoma is dependent on silencing of the $O^{6}$-methylguanine-DNA methyltransferase (MGMT) DNA repair gene (22). However, MGMT gene silencing may also occur via promoter methylation in soft tissue sarcomas, although this is rare (23). The ability of alkylating agents to induce apoptosis is dependent not only on MGMT activity, but also on the activity of various survival pathways $(24,25)$, and the mechanism to induce the apoptosis 

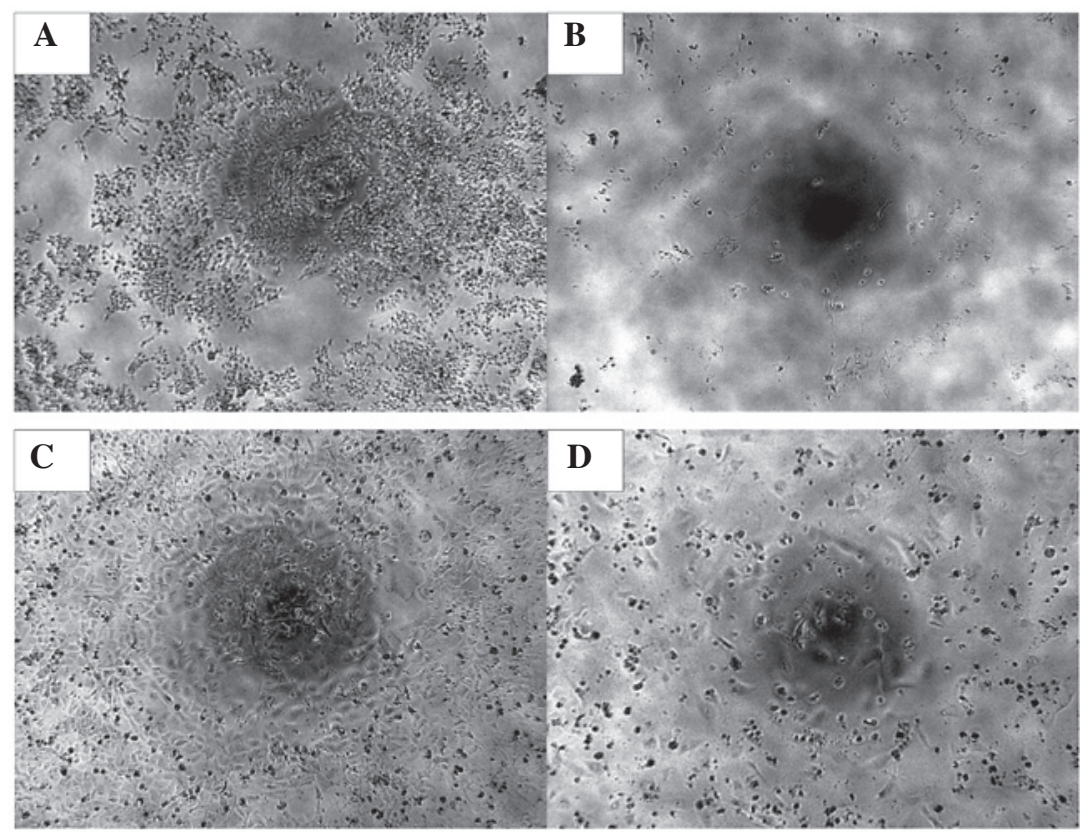

Figure 2. Typical morphological changes in MSS cells following TMZ exposure, observed using a phase-contrast microscope $120 \mathrm{~h}$ post TMZ exposure. Distinct cell death observed in SKNMC cells following treatment with (A) $10 \mu \mathrm{M} \mathrm{TMZ}$ and (B) $1000 \mu \mathrm{M}$ of TMZ. Such cell death was also induced in ten of the other cell lines (not shown). No cell lysis was observed in FU-EPS-1 cells following (C) $10 \mu \mathrm{M} \mathrm{TMZ}$ and (D) $1000 \mu \mathrm{M}$ TMZ; or ASPS-KY cells (not shown). Magnification, x100. TMZ, temozolomide; MSS, musculoskeletal sarcoma.
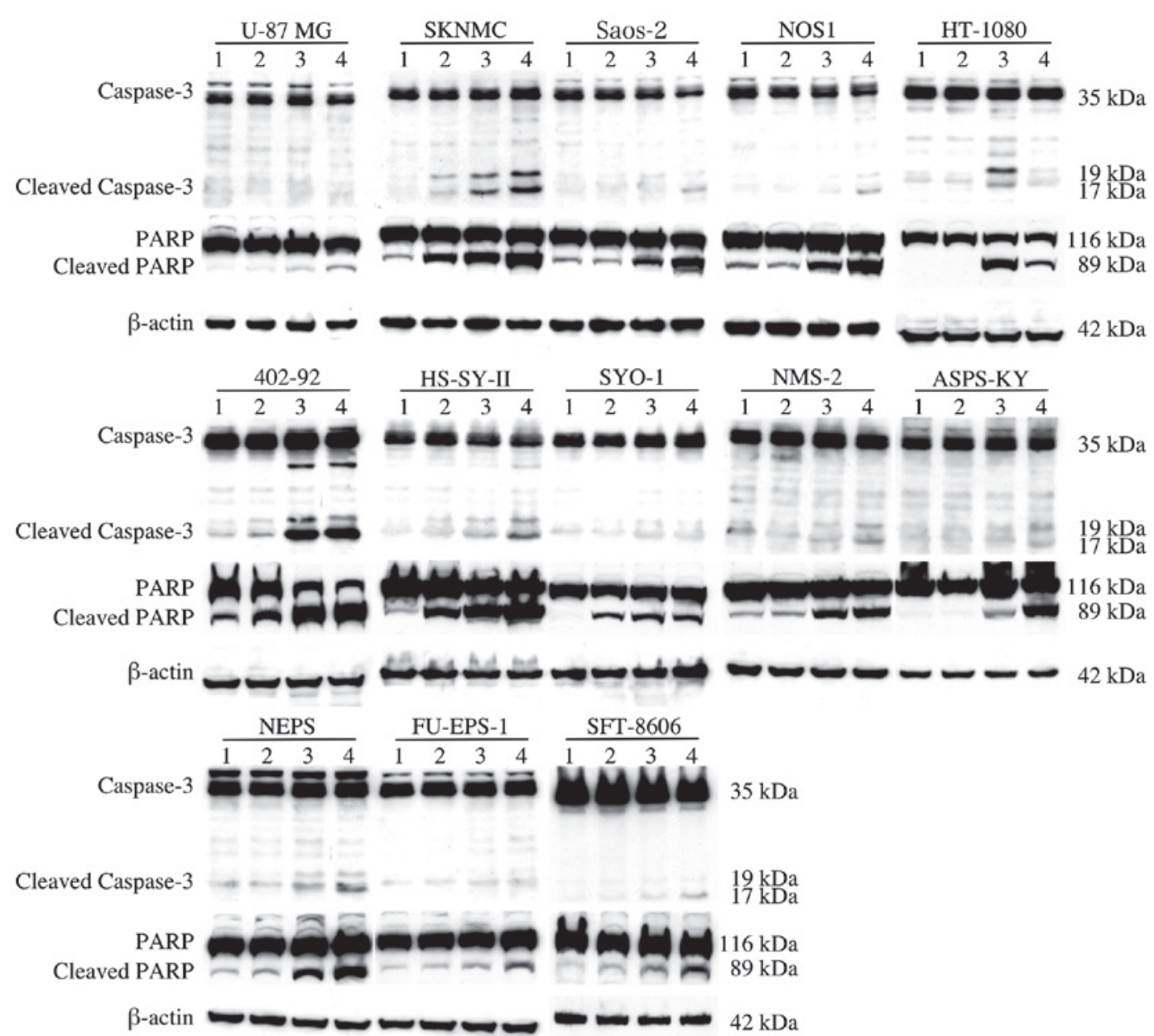

Figure 3. Western blot analysis of apoptotic proteins. Cleavage of caspase-3 has a critical role in apoptosis, and the proteolytic cleavage of PARP by activated caspase- 3 serves as a marker of apoptosis. Apoptosis was induced by TMZ in all tested cell lines. Marked activation of caspase- 3 protein was detected at 48 or $72 \mathrm{~h}$ following exposure to TMZ in SKNMC, Saos-2, NOS1, HS-SY-II, HT-1080, 402-92 and NEPS cells. Lanes: 1, control; 2, treated with TMZ for 24 h; 3 , treated with TMZ for $48 \mathrm{~h} ; 4$, treated with TMZ for $72 \mathrm{~h}$. TMZ, temozolomide; PARP, poly adenosine diphosphate ribose polymerase. 


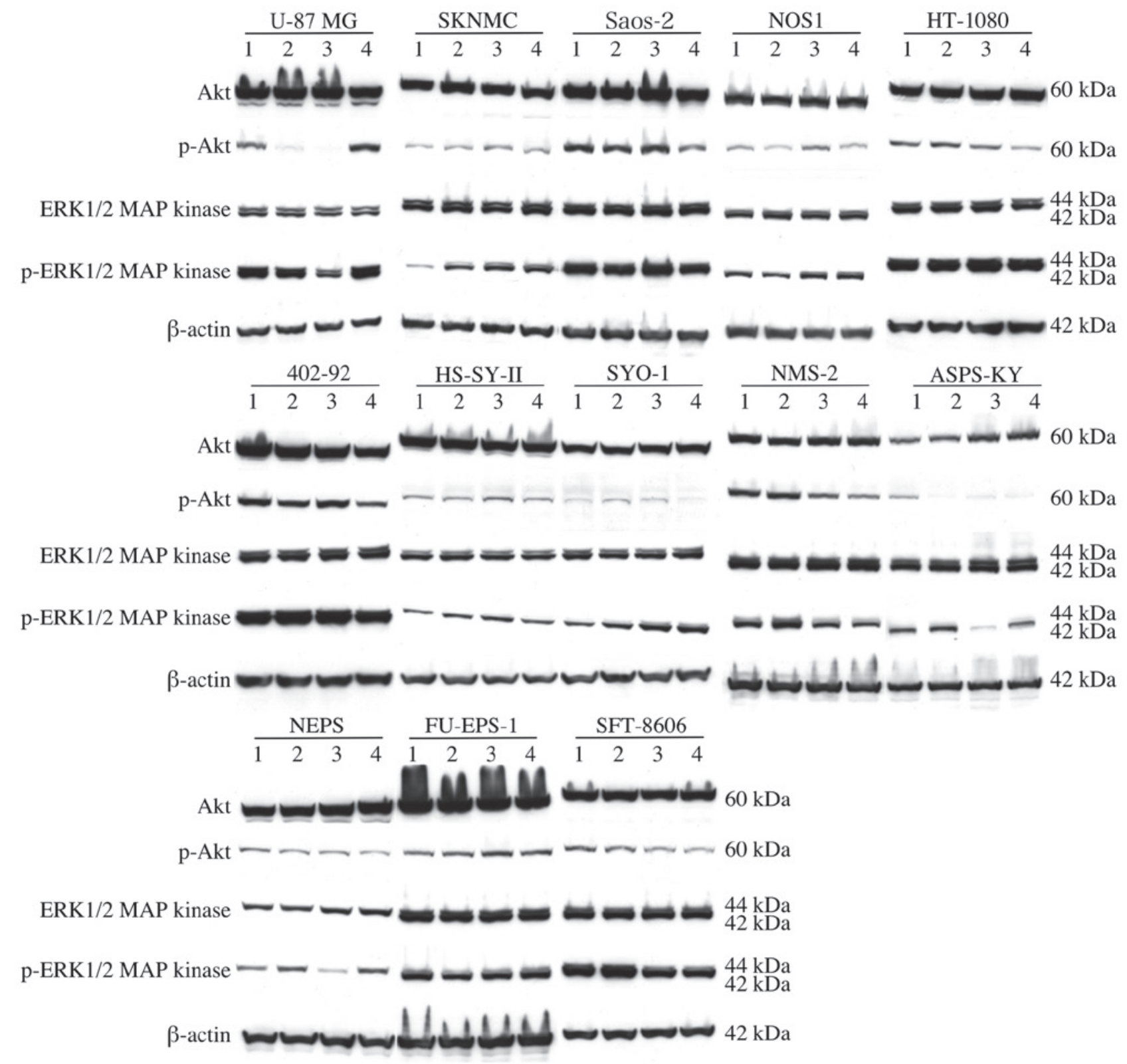

Figure 4. Western blot analysis of cell survival/proliferation pathways. PI3K/Akt and ERK1/2 MAPK are associated with cell survival and proliferation. The phosphorylation status of PI3K/Akt and ERK1/2 MAPK was evaluated by western blotting. Basal levels of p-Akt were reduced in a time-dependent manner in SYO-1, HT-1080, NMS-2, and ASPS-KY cells following treatment with $250 \mu \mathrm{M}$ TMZ. By contrast, the expression of p-ERK1/2 was 2 was marginally decreased in ASPS-KY and NEPS cells. Lanes: 1, control; 2, treated with TMZ for 24 h; 3, treated with TMZ for 48 h; 4, treated with TMZ for 72 h. TMZ, temozolomide; PI3K, phosphoinositide 3-kinase; ERK1/2, extracellular signal-regulated kinase 1/2; MAPK, mitogen activated protein kinase.

in MSS may differ from in glioblastoma. Therefore, analysis of the pro-apoptotic effects of TMZ, as well as the study of interactions between TMZ and anti-apoptotic/proliferation pathways (PI3K/Akt and ERK1/2 MAPK), may contribute to improving MSS patient survival.

The results of the present study demonstrated that TMZ dose-dependently reduced the viability of MSS cells by inducing apoptosis, with a parallel increase in caspase-3 and PARP activity. An XTT assay was used to compare the sensitivity of TMZ of treated MSS cells and U-87 MG cells to TMZ (26,27). The $\mathrm{IC}_{50}$ values of the MSS cells following $120 \mathrm{~h}$ of treatment ranged from 72 to $1167 \mu \mathrm{M}$, whereas the $\mathrm{IC}_{50}$ value in U-87 MG glioblastoma cells was $348 \mu \mathrm{M}$. In 5 of the $12 \mathrm{MSS}$ cell lines tested, the $\mathrm{IC}_{50}$ value was lower than that of U-87
MG cells. TMZ treatment was also associated with marked morphological changes, including cellular floating and cell debris accumulation following $120 \mathrm{~h}$ treatment with $1000 \mu \mathrm{M}$ TMZ. Although TMZ induced apoptosis in all cell lines evaluated, the sensitivity of these sarcomas to TMZ varied. These findings regarding cell viability suggest that TMZ treatment may be effective in certain types of MSS.

The mechanism of TMZ action remains controversial. Hirose et al (28) reported that, in glioma cells, TMZ treatment induced low levels of apoptosis compared with those observed in lymphoid cells. In addition, TMZ was shown to induce cell cycle arrest in the G2/M phase. By contrast, Kanzawa et al (29) demonstrated that TMZ induced autophagy, but not apoptosis, in malignant glioma cells. Furthermore, Roos et al (27) 
indicated that cell death induced by TMZ in gliomas was due to apoptosis. In the present study, TMZ was demonstrated to significantly increase the activation of apotosis-associated proteins caspase-3 and PARP. This induction of apoptosis may explain the reduction in MSS cell proliferation observed in the presence of TMZ, and may constitute a novel apoptotic mechanism in these cells.

The PI3K/Akt and ERK1/2 MAPK signaling pathways are significant in protein synthesis, cell proliferation, angiogenesis and metabolism (30). Therefore, the phosphorylation of these signaling pathway components was also analyzed in the present study. The results indicated that PI3K/Akt was constitutively active in MSS cells, and that this activation was suppressed in certain cells and maintained in others following treatment with TMZ. Notably, p-Akt markedly increased at $72 \mathrm{~h}$, indicating possible re-activation of $\mathrm{p}$-Akt. The data also revealed that ERK1/2 MAPK activation was marginally decreased in certain cell types. Combined, these results indicated that MSS cells are characterized by aberrant activation of these signaling pathways, an effect which is maintained or suppressed in the presence of TMZ. TMZ decreased the viability of certain cell lines. The viability of all cell lines only decreased in very high concentrations of TMZ. Therefore, it is considered that certain MSS cells were resistant while some were relatively sensitive to TMZ. Furthermore, these observations emphasize the plasticity of MSS cells and their ability to resist chemotherapy by blocking the induction of apoptosis. This plasticity may explain the resistance of some MSS cells to TMZ, as well as the differences in MSS cell sensitivity to alkylating agents. These results also suggested that combinations of traditional chemotherapy with molecular-based therapy, in particular therapy associated with signaling pathways, may be used to effectively treat MSS. The methylation status of the MGMT promoter in MSS cells was not determined in the present study, and additional experiments may be required to clarify whether there is an association between MGMT status and MSS cell sensitivity to TMZ.

Though the apoptotic signaling pathways induced during TMZ treatment remain elusive, understanding the mechanisms involved in the induction of apoptosis may contribute to improving the prognosis of patients with MSS. More detailed investigations are needed to clarify whether TMZ presents a promising strategy for MSS therapy or not.

\section{References}

1. Gottlieb JA, Benjamin RS, Baker LH, et al: Role of DTIC (NSC-45388) in the chemotherapy of sarcomas. Cancer Treat Rep 60: 199-203, 1976.

2. Stevens MF, Hickman JA, Langdon SP, et al: Antitumor activity and pharmacokinetics in mice of 8-carbamoyl-3-methyl-imidazo [5,1-d]-1,2,3,5-tetrazin-4(3H)-one (CCRG 81045; M \& B 39831), a novel drug with potential as an alternative to dacarbazine. Cancer Res 47: 5846-5852, 1987.

3. Siegel R, Ma J, Zou Z and Jemal A: Cancer statistics, 2014. CA Cancer J Clin 64: 9-29, 2014.

4. Moreira-Gonzalez A, Djohan R and Lohman R: Considerations surrounding reconstruction after resection of musculoskeletal sarcomas. Cleve Clin J Med 77 (Suppl 1): S18-S22, 2010.

5. Trent JC, Beach J, Burgess MA, et al: A two-arm phase II study of temozolomide in patients with advanced gastrointestinal stromal tumors and other soft tissue sarcomas. Cancer 98: 2693-2699, 2003.
6. Garcia del Muro X, Lopez-Pousa A, Martin J, Buesa JM, Martinez-Trufero J, Casado A, Poveda A, Cruz J, Bover I and Maurel J; Spanish Group for Research on Sarcomas: A phase II trial of temozolomide as a 6-week, continuous, oral schedule in patients with advanced soft tissue sarcoma: A study by the Spanish Group for Research on Sarcomas. Cancer 104: 1706-1712, 2005.

7. Stacchiotti S, Tortoreto M, Bozzi F, et al: Dacarbazine in solitary fibrous tumor: A case series analysis and preclinical evidence vis-a-vis temozolomide and antiangiogenics. Clin Cancer Res 19: 5192-5201, 2013.

8. Casey DA, Wexler LH, Merchant MS, Chou AJ, Merola PR, Price AP and Meyers PA: Irinotecan and temozolomide for Ewing sarcoma: The Memorial Sloan-Kettering experience. Pediatr Blood Cancer 53: 1029-1034, 2009.

9. Raciborska A, Bilska K, Drabko K, Chaber R, Pogorzala M, Wyrobek E, Polczyńska K, Rogowska E, Rodriguez-Galindo C and Wozniak W: Vincristine, irinotecan and temozolomide in patients with relapsed and refractory Ewing sarcoma. Pediatr Blood Cancer 60: 1621-1625, 2013.

10. Hotta T, Motoyama T and Watanabe H: Three human osteosarcoma cell lines exhibiting different phenotypic expressions. Acta Pathol Jpn 42: 595-603, 1992.

11. Imaizumi S, Motoyama T, Ogose A, Hotta T and Takahashi HE: Characterization and chemosensitivity of two human malignant peripheral nerve sheath tumour cell lines derived from a patient with neurofibromatosis type 1. Virchows Arch 433: 435-441, 1998.

12. Hoshino M, Kawashima H, Ogose A, et al: Serum CA 125 expression as a tumor marker for diagnosis and monitoring the clinical course of epithelioid sarcoma. J Cancer Res Clin Oncol 136: 457-464, 2010.

13. Sonobe H, Manabe Y, Furihata M, Iwata J, Oka T, Ohtsuki Y, Mizobuchi H, Yamamoto H, Kumano O and Abe S: Establishment and characterization of a new human synovial sarcoma cell line, HS-SY-II. Lab Invest 67: 498-505, 1992.

14. Kawai A, Naito N, Yoshida A, et al: Establishment and characterization of a biphasic synovial sarcoma cell line, SYO-1. Cancer Lett 204: 105-113, 2004.

15. Aman P, Ron D, Mandahl N, et al: Rearrangement of the transcription factor gene CHOP in myxoid liposarcomas with $\mathrm{t}(12 ; 16)(\mathrm{q} 13 ; \mathrm{p} 11)$. Genes Chromosomes Cancer 5: 278-285, 1992.

16. Hoshino M, Ogose A, Kawashima H, et al: Molecular analyses of cell origin and detection of circulating tumor cells in the peripheral blood in alveolar soft part sarcoma. Cancer Genet Cytogenet 190: 75-80, 2009.

17. Kusakabe H, Iwasaki H, Sano K and Kiyokane K: Expression of lung resistance protein in epithelioid sarcoma in vitro and in vivo. Arch Dermatol Res 292: 292-300, 2000.

18. Fernandes-Alnemri T, Litwack G and Alnemri ES: CPP32, a novel human apoptotic protein with homology to Caenorhabditis elegans cell death protein Ced-3 and mammalian interleukin-1 beta-converting enzyme. J Biol Chem 269: 30761-30764, 1994.

19. Oliver FJ, de la Rubia G, Rolli V, et al: Importance of poly (ADP-ribose) polymerase and its cleavage in apoptosis. Lesson from an uncleavable mutant. J Biol Chem 273: 33533-33539, 1998.

20. Liu H, Zhou L, Shi S, et al: Oligosaccharide G19 inhibits U-87 MG human glioma cells growth in vitro and in vivo by targeting epidermal growth factor (EGF) and activating p53/p21 signaling. Glycobiology 24: 748-765, 2014.

21. Wu L, Yang L, Xiong Y, et al: Annexin A5 promotes invasion and chemoresistance to temozolomide in glioblastoma multiforme cells. Tumour Biol 35: 12327-12337, 2014.

22. Hegi ME, Diserens AC, Gorlia T, Hamou MF, de Tribolet N, Weller M, Kros JM, Hainfellner JA, Mason W, Mariani L, et al: MGMT gene silencing and benefit from temozolomide in glioblastoma. N Engl J Med 352: 997-1003, 2005.

23. Jakob J, Hille M, Sauer C, Ströbel P, Wenz F and Hohenberger P: O6-methylguanine-DNA methyltransferase (MGMT) promoter methylation is a rare event in soft tissue sarcoma. Radiat Oncol 7: $180,2012$.

24. De Salvo M, Maresca G, D'agnano I, et al: Temozolomide induced c-Myc-mediated apoptosis via Akt signalling in MGMT expressing glioblastoma cells. Int J Radiat Biol 87: 518-533, 2011.

25. Wang H, Cai S, Ernstberger A, Bailey BJ, Wang MZ, Cai W, Goebel WS, Czader MB, Crean C, Suvannasankha A, et al: Temozolomide-mediated DNA methylation in human myeloid precursor cells: Differential involvement of intrinsic and extrinsic apoptotic pathways. Clin Cancer Res 19: 2699-2709, 2013. 
26. Kanzawa T, Germano IM, Kondo Y, Ito H, Kyo S and Kondo S: Inhibition of telomerase activity in malignant glioma cells correlates with their sensitivity to temozolomide. Br J Cancer 89: 922-929, 2003.

27. Roos WP, Batista LF, Naumann SC, Wick W, Weller M, Menck CF and Kaina B: Apoptosis in malignant glioma cells triggered by the temozolomide-induced DNA lesion $\mathrm{O}_{6}$-methylguanine. Oncogene 26: 186-197, 2007.

28. Hirose Y, Berger MS and Pieper RO: p53 effects both the duration of $\mathrm{G} 2 / \mathrm{M}$ arrest and the fate of temozolomide-treated human glioblastoma cells. Cancer Res 61: 1957-1963, 2001.
29. Kanzawa T, Germano IM, Komata T, Ito H, Kondo Y and Kondo S: Role of autophagy in temozolomide-induced cytotoxicity for malignant glioma cells. Cell Death Differ 11: 448-457, 2004.

30. Mahalingam D, Mita A, Sankhala K, Swords R, Kelly K, Giles F and Mita MM: Targeting sarcomas: Novel biological agents and future perspectives. Curr Drug Targets 10: 937-949, 2009. 